\title{
SINGULAR INTEGRAL OPERATORS ASSOCIATED TO CURVES WITH RATIONAL COMPONENTS
}

\author{
MAGALI FOLCH-GABAYET AND JAMES WRIGHT
}

Abstract. We prove $L^{p}\left(\mathbb{R}^{n}\right), 1<p<\infty$, bounds for

$$
H f(x)=p . v . \int_{-\infty}^{\infty} f\left(x_{1}-R_{1}(t), \ldots, x_{n}-R_{n}(t)\right) d t / t
$$

and

$$
M f(x)=\sup _{h>0} \frac{1}{h} \int_{0}^{h}\left|f\left(x_{1}-R_{1}(t), \ldots, x_{n}-R_{n}(t)\right)\right| d t
$$

where $R_{j}(t)=P_{j}(t) / Q_{j}(t), j=1,2, \ldots, n$, are rational functions. Our bounds depend only on the degrees of the polynomials $P_{j}, Q_{j}$ and, in particular, they do not depend on the coefficients of these polynomials.

\section{INTRODUCTION}

To any curve in $\mathbb{R}^{n}$ parametrised by $\Gamma: \mathbb{R} \rightarrow \mathbb{R}^{n}$, we associate a maximal operator

$$
M f(x)=\sup _{h>0} \frac{1}{h} \int_{0}^{h}|f(x-\Gamma(t))| d t
$$

and a singular integral operator

$$
H f(x)=p . v \cdot \int_{-\infty}^{\infty} f(x-\Gamma(t)) d t / t
$$

defined initially on test functions $f \in C_{c}^{\infty}\left(\mathbb{R}^{n}\right)$. These operators have a long history (see, e.g., [5] and [6]) and many $L^{p}$ estimates have been obtained for various classes of curves. For example, it is well known that $M$ and $H$ are bounded on $L^{p}\left(\mathbb{R}^{n}\right), 1<p<\infty$, whenever $\Gamma$ is a polynomial curve; that is, the components of $\Gamma(t)=\left(P_{1}(t), \ldots, P_{n}(t)\right)$ are polynomials, and furthermore, these estimates are independent of the coefficients, depending only on the degrees of the polynomials (see, e.g., [6]).

In this paper we extend this result to curves with rational components.

Theorem 1.1. Let $\Gamma(t)=\left(R_{1}(t), \ldots, R_{n}(t)\right)$ where $R_{j}=P_{j} / Q_{j}, j=1, \ldots, n$, are rational functions. Then the associated maximal and singular integral operators

Received by the editors October 19, 2004 and, in revised form, May 30, 2006.

2000 Mathematics Subject Classification. Primary 42B15.

The first author acknowledges financial support from CONACyT (37046-E) and DGAPAUNAM (PAPIIT IN101303).

The second author would like to thank the warm hospitality of the Instituto de Matemáticas, Universidad Nacional Autónoma de México where most of the research for this paper was conducted. 
$M$ and $H$ are bounded on $L^{p}\left(\mathbb{R}^{n}\right), 1<p<\infty$, with bounds independent of the coefficients of the polynomials $P_{j}, Q_{j}$.

Remarks 1.2.

- In [3], Theorem 1.1 was established in two dimensions $n=2$ when $R_{1}(t)=t$ and $R_{2}$ is an arbitrary rational function (i.e., in the case where the curve is a graph of a rational function).

- For a general curve $\Gamma$ the principal-value integral defining $H$ may not exist, even for $f \in C_{c}^{\infty}\left(\mathbb{R}^{n}\right)$. However it is easy to check that when the components of $\Gamma$ are rational functions, the principal-value integral does indeed exist whenever $f \in C_{c}^{\infty}\left(\mathbb{R}^{n}\right)$.

In the next section we will outline the ideas which go into the proof of Theorem 1.1. In section 3 we state and prove a series of preliminary lemmas needed for the proof of Theorem 1.1. In section 4 we establish the basic reduction for the operators $H$ and $M$, as outlined in section 2, which allows us to carry out the proof of the $L^{p}$ boundedness of $H$ and $M$ in the final section.

Notation: Let $A, B$ be complex-valued quantities. We use the notation $A \lesssim B$ or $A=O(B)$ to denote the estimate $|A| \leq C|B|$ where $C$ depends only on $n$ and the degrees of the polynomials defining the curve $\Gamma$. We use $A \sim B$ to denote the estimates $A \lesssim B \lesssim A$.

\section{IDEA OF PROOF FOR THEOREM 1.1}

We illustrate the ideas for the singular integral operator $H$. By factoring a polynomial $P(t)$ into its linear factors, it is easy to see that outside a bounded number of "dyadic" intervals, $P$ behaves like various monomials on the complementary intervals (see Lemma 3.1 below). Hence we can reduce ourselves to bounding

$$
H_{G} f(x)=\int_{|t| \in G} f(x-\Gamma(t)) d t / t
$$

where $G$ is an interval (possibly very long) on which each rational function $R_{m}=$ $P_{m} / Q_{m}$ defining $\Gamma$ behaves like $c t^{j_{m}-k_{m}}$ for some nonnegative integers $j_{m}, k_{m} \geq 0$. We decompose

$$
\begin{aligned}
H_{G} f(x) & =\sum_{\ell} \int_{|t| \in G \cap\left[2^{\ell}, 2^{\ell+1}\right]} f(x-\Gamma(t)) d t / t \\
& =\sum_{\ell} \int_{|t| \in 2^{-\ell} G \cap[1,2]} f\left(x-\Gamma\left(2^{\ell} t\right)\right) d t / t
\end{aligned}
$$

where the last sum can be written as $\sum_{\ell \in \mathbb{Z}} f * \sigma_{\ell}^{(\ell)}(x)$. Here the "normalised" measures $\sigma^{(\ell)}$ are defined on a test function $\phi$ by

$$
\left\langle\sigma^{(\ell)}, \phi\right\rangle=\int_{|t| \in 2^{-\ell} G \cap[1,2]} \phi\left(R_{1, \ell}(t), \ldots, R_{n, \ell}(t)\right) d t / t,
$$

where $R_{m, \ell}(t)=2^{-\left(j_{m}-k_{m}\right) \ell} R_{m}\left(2^{\ell} t\right), m=1, \ldots, n$, are "normalised" functions. The measures $\sigma_{\ell}^{(\ell)}$ are $2^{\ell}$ dilates of $\sigma^{(\ell)}$, given by the 1-parameter dilations $2^{\ell} \circ x=$ $\left(2^{\left(j_{1}-k_{1}\right) \ell} x_{1}, \ldots, 2^{\left(j_{n}-k_{n}\right) \ell} x_{n}\right)$. At this point we would like to appeal to a special 
instance of a general result of Ricci and Stein [4] giving sufficient conditions which guarantee $L^{p}, 1<p<\infty$, boundedness for singular integral operators of the form

$$
f \mapsto \sum_{\ell \in \mathbb{Z}} f * \sigma_{\ell}^{(\ell)} .
$$

The family $\left\{\sigma^{(\ell)}\right\}$ is required to satisfy three conditions. First, there is a cancellation condition; besides requiring that each $\sigma^{(\ell)}$ have mean zero, the Fourier transform of $\sigma^{(\ell)}$ should also vanish on the subspace $V=\left\{\xi \in \mathbb{R}^{n}: \xi=\sum_{m \in S} \xi_{m} e_{m}\right\}$ where $S=\left\{m: j_{m}-k_{m}=0\right\}$. However for $\sigma^{(\ell)}$ defined in (2),

$$
\widehat{\sigma^{(\ell)}}(\xi)=\int_{|s| \in 2^{-\ell} G \cap[1.2]} e^{i\left[\xi_{1} R_{1, \ell}(s)+\cdots+\xi_{n} R_{n, \ell}(s)\right]} d s / s
$$

vanishes only at 0 in general. Hence we would like $j_{m} \neq k_{m}$ for each $m=1,2, \ldots, n$.

The second condition is a uniform regularity condition:

$$
\left|\widehat{\sigma^{(\ell)}}(\xi)\right| \leq C(1+|\xi|)^{-\epsilon}
$$

for some $C, \epsilon>0$ independent of $\ell \in \mathbb{Z}$. Now $\sigma^{(\ell)}$ is a perturbation of a measure supported on an orbit of the dilation group $\delta \circ x=\left(\delta^{j_{1}-k_{1}} x_{1}, \ldots, \delta^{j_{n}-k_{n}} x_{n}\right)$. In [4], Lemma 6.1 gives a necessary and sufficient condition for a measure supported along an orbit to satisfy the decay estimate (3); namely, the exponents $\left\{j_{m}-k_{m}\right\}$ must be nonzero and distinct. Hence we would not only like the numbers $\left\{j_{m}-k_{m}\right\}$ to be nonzero but also distinct. We will be able to achieve this by using the fact that the $L^{p}$ operator norm of $H_{G}$ is invariant under conjugation by the group of invertible affine transformations. That is, we may replace the rational functions $\Gamma=\left(R_{1}, \ldots, R_{n}\right)$ by $A \Gamma+\vec{v}$, where $A$ is a constant invertible $n \times n$ matrix and $\vec{v}$ is a constant vector in $\mathbb{R}^{n}$, without changing the operator norm of $H_{G}$. We will describe a procedure in section 4 below, using affine transformations, to reduce us to bounding $H_{G}$ where now $R_{m}(t) \sim c t^{j_{m}-k_{m}}$ on $G$ and $\left\{j_{m}-k_{m}\right\}$ are nonzero, distinct numbers. We will then be able to go on to establish the decay estimates (3), putting us in a position to use the result of Ricci and Stein on $L^{p}$ boundedness of singular integral operators of the form $f \mapsto \sum_{\ell} f * \sigma_{\ell}^{(\ell)}$.

However there is a third condition that the family $\left\{\sigma^{(\ell)}\right\}$ is required to satisfy which can be viewed as a further regularity condition. It is that there is a finite measure $\nu$ so that $\left|\sigma^{(\ell)}\right| \leq \nu$ for all $\ell \in \mathbb{Z}$, and unfortunately, this condition is not satisfied in general by the $\sigma^{(\ell)}$ defined in (2). Nevertheless our situation is a very special case of the Ricci-Stein multi-parameter theory, being generated only by a 1-parameter group of dilations, albeit the exponents $\left\{j_{m}-k_{m}\right\}$ can be positive or negative. Instead, fortunately, we will be able to employ the results of Duoandikoetxea and Rubio de Francia in [2] to obtain the $L^{p}$ bounds for $H_{G}$ and hence establish Theorem 1.1 in full generality for $H$. Similar ideas successfully bound the maximal function $M$.

\section{Preliminaries}

The following lemma is a simple variant of a lemma in [1].

Lemma 3.1. Let $P(t)=D \prod_{j=1}^{d}\left(t-z_{j}\right)=\sum_{k=0}^{d} p_{k} t^{k}$ be a polynomial of degree $d$ whose roots are ordered so that $\left|z_{1}\right| \leq \ldots \leq\left|z_{d}\right|$. Then there exists a $C=C(d)$ 
such that for any $A \geq C(d)$ and $1 \leq j \leq d$, if $G=\left[A\left|z_{j}\right|, A^{-1}\left|z_{j+1}\right|\right]$ is nonempty $\left(G=\left[A\left|z_{d}\right|, \infty\right)\right.$ if $\left.j=d\right)$,

i) $P(t) \sim p_{j} t^{j} \quad$ for $|t| \in G$;

ii) $p_{j} \sim D \prod_{\ell=j+1}^{d} z_{\ell}$, and in particular $p_{j} \neq 0$;

iii) if $k<j, \quad p_{k} \lesssim p_{j} \prod_{\ell=k+1}^{j} z_{\ell} ; \quad$ and if $j<k, \quad p_{k} \lesssim p_{j} \prod_{\ell=j+1}^{k} z_{\ell}{ }^{-1}$.

Proof. Clearly for $|t| \in G$ (and any $A>1$ ),

$$
(1-1 / A)^{d}|D|\left[\prod_{\ell=j+1}^{d}\left|z_{\ell}\right|\right] \leq|P(t)| /|t|^{j} \leq(1+1 / A)^{d}|D|\left[\prod_{\ell=j+1}^{d}\left|z_{\ell}\right|\right],
$$

which shows that i) follows from ii). To establish ii) we write

$$
\begin{aligned}
p_{j} & =(-1)^{j} D \sum_{\ell_{1}<\cdots<\ell_{d-j}} z_{\ell_{1}} \cdots z_{\ell_{d-j}} \\
& =(-1)^{j} D \sum_{\substack{\ell_{1}<\ldots<\ell_{d-j} \\
\ell_{1} \leq j}} z_{\ell_{1}} \cdots z_{\ell_{d-j}}+(-1)^{j} D z_{j+1} \cdots z_{d} \\
& =I \quad+I I,
\end{aligned}
$$

and hence since $\left|z_{\ell}\right| \leq(1 / A)\left|z_{\ell^{\prime}}\right|$ whenever $\ell \leq j \leq \ell^{\prime}-1$,

$$
A|I| \lesssim|D|\left|z_{j+1}\right| \cdots\left|z_{d}\right|=|I I| .
$$

Therefore if $A \gtrsim 1$ is large enough,

$$
p_{j} \sim I I=D \prod_{\ell=j+1}^{d} z_{\ell},
$$

establishing ii) and hence i). From the formula

$$
p_{k}=(-1)^{k} D \sum_{\ell_{1}<\cdots<\ell_{d-k}} z_{\ell_{1}} \cdots z_{\ell_{d-k}}
$$

we see by ii) that if $k<j$,

$$
p_{k} \lesssim D \prod_{\ell=k+1}^{d} z_{\ell} \sim p_{j} \prod_{\ell=k+1}^{j} z_{\ell}
$$

and if $j<k$,

$$
p_{k} \lesssim D \prod_{\ell=k+1}^{d} z_{\ell} \sim p_{j} \prod_{\ell=j+1}^{k} z_{\ell}^{-1} .
$$

This completes the proof of the lemma.

Remark 3.2. Lemma 3.1, part i), shows that with respect to $P, \mathbb{R}^{+}$can be decomposed as

$$
\mathbb{R}^{+}=\bigcup_{\ell=1}^{M} G_{\ell} \cup \bigcup_{\ell=1}^{M-1} D_{\ell}
$$

into disjoint intervals $(M=O(1))$ which depend on the choice of $A$ where the $D_{\ell}$ are dyadic in the sense that if $D_{\ell}=[a, b)$, then $b / a=O(1)$. On the complementary intervals $G_{\ell}$ (which we call "gaps"), if $|t| \in G_{\ell}, P(t) \sim p_{j_{\ell}} t^{j_{\ell}}$ for some $j_{\ell} \geq 0$ (and of course $\left.p_{j_{\ell}} \neq 0\right)$. See [1]. 
For a rational function $R=P / Q$, where $P(t)=D \prod_{\ell=1}^{d_{p}}\left(t-z_{\ell}\right), Q(t)=$ $E \prod_{\ell=1}^{d_{q}}\left(t-w_{\ell}\right)$ with $\left|z_{1}\right| \leq \cdots \leq\left|z_{d_{p}}\right|$ and $\left|w_{1}\right| \leq \cdots \leq\left|w_{d_{q}}\right|$, Lemma 3.1 tells us that $R(t) \sim\left[p_{j} / q_{k}\right] t^{j-k}$ on a gap $G=\left[A\left|z_{j}\right|, A^{-1}\left|z_{j+1}\right|\right] \cap\left[A\left|w_{k}\right|, A^{-1}\left|w_{k+1}\right|\right]$, if $A \gtrsim 1$ is large enough. We now examine higher derivatives of $R$ on $G$ in the following two lemmas. We begin with the case $j \geq k$.

Lemma 3.3. Let $R=P / Q$ be a rational function and $G$ a gap as described above. Then for any integer $n \geq 0, A \gtrsim C_{n}$ can be chosen large enough so that on $G$, if $j \geq k$,

$$
R^{(n)}(t)=R(t)\left[\sum_{k+1 \leq \ell_{1} \neq \cdots \neq \ell_{n} \leq j} \prod_{m=1}^{n} \frac{1}{t-z_{\ell_{m}}}+E_{n}(t)\right]
$$

where $\left|(d / d t)^{r} E_{n}(t)\right| \lesssim C_{n, r} A^{-1}|t|^{-n-r}$ on $G$ for all $r \geq 0$.

Proof. We begin with the case $n=1$.

$$
\begin{aligned}
R^{\prime}(t) & =R(t)\left[P^{\prime}(t) / P(t)-Q^{\prime}(t) / Q(t)\right] \\
& =R(t)\left[\sum_{\ell=1}^{d_{p}} \frac{1}{t-z_{\ell}}-\sum_{\ell=1}^{d_{q}} \frac{1}{t-w_{\ell}}\right] .
\end{aligned}
$$

We make the following two simple observations on $G$ :

$$
\left|\frac{1}{t-z_{\ell}}\right|,\left|\frac{1}{t-w_{\ell^{\prime}}}\right| \leq C[A|t|]^{-1}, \quad \ell>j, \ell^{\prime}>k
$$

and

$$
\left|\frac{1}{t-z_{\ell}}-\frac{1}{t-w_{\ell^{\prime}}}\right|=\frac{\left|z_{\ell}-w_{\ell^{\prime}}\right|}{\left|t-z_{\ell}\right|\left|t-w_{\ell^{\prime}}\right|} \leq C[A|t|]^{-1}, \quad \ell, \ell^{\prime} \leq k
$$

Hence

$$
R^{\prime}=R\left[\sum_{\ell=k+1}^{j} \frac{1}{t-z_{\ell}}+E_{1}(t)\right]
$$

where

$$
E_{1}(t)=\sum_{\ell=1}^{k}\left[\frac{1}{t-z_{\ell}}-\frac{1}{t-w_{\ell}}\right]+\sum_{\ell=j+1}^{d_{p}} \frac{1}{t-z_{\ell}}-\sum_{\ell=k+1}^{d_{q}} \frac{1}{t-w_{\ell}}
$$

satisfies $\left|E_{1}^{(r)}(t)\right| \leq C A^{-1}|t|^{-r-1}, \forall r \geq 0$ on $G$ by (5) and (6), establishing (4) when $n=1$.

The proof now proceeds by induction on $n$; if (4) holds for derivatives up to order $n-1$,

$$
\begin{gathered}
R^{(n)}(t)=R^{\prime}(t)\left[\sum_{k+1 \leq \ell_{1} \neq \cdots \neq \ell_{n-1} \leq j} \prod_{m=1}^{n-1} \frac{1}{t-z_{\ell_{m}}}+E_{n-1}(t)\right] \\
+R(t)\left[-\sum_{r=1}^{n-1} \sum_{k+1 \leq \ell_{1} \neq \cdots \neq \ell_{n-1} \leq j} \frac{1}{\left(t-z_{\ell_{1}}\right)} \cdots \frac{1}{\left(t-z_{\ell_{r}}\right)^{2}} \cdots \frac{1}{\left(t-z_{\ell_{n-1}}\right)}\right. \\
\left.+E_{n-1}^{\prime}(t)\right]
\end{gathered}
$$




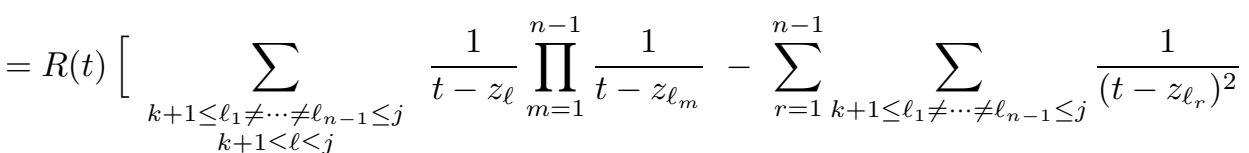

$$
\begin{aligned}
& \left.\times \prod_{\substack{1 \leq m \leq n-1 \\
m \neq r}} \frac{1}{\left(t-z_{\ell_{m}}\right)}+E_{n}(t)\right]
\end{aligned}
$$

where

$E_{n}(t)=E_{n-1}(t)\left(\sum_{\ell=k+1}^{j} \frac{1}{t-z_{\ell}}+E_{1}(t)\right)+E_{1}(t) \sum_{k+1 \leq \ell_{1} \neq \cdots \neq \ell_{n-1} \leq j} \prod_{m=1}^{n-1} \frac{1}{t-z_{\ell}}+E_{n-1}^{\prime}(t)$

is easily seen to satisfy the derivative bounds on $G$, proving (4) for general $n$.

Remarks 3.4.

- The sum

$$
\sum_{k+1 \leq \ell_{1} \neq \cdots \neq \ell_{n} \leq j} \prod_{m=1}^{n} \frac{1}{t-z_{\ell_{m}}}
$$

in (4) is interpreted as zero when $j-k \leq n-1$.

- It will be important for us to keep track of the number of terms in the sum (4) which in this case is $(j-k)(j-k-1) \ldots(j-k-n+1)$. This shows in particular that the sum is empty if $j-k \leq n-1$.

We now turn to the case $j<k$ which unfortunately is somewhat more involved. As in the case $k \leq j$, it will be important for us to keep track of the number of terms in various sums. To this end we associate to every strictly positive multiindex $\alpha=\left(\alpha_{1}, \ldots, \alpha_{r}\right), \alpha_{i}>0,1 \leq i \leq r$ a size $|\alpha|=\alpha_{1}+\cdots+\alpha_{r}$ and a length $l(\alpha)=r$.

Lemma 3.5. Let $R=P / Q$ and $G$ be as in Lemma 3.3 but where now $j<k$. For any integer $n \geq 1, A \gtrsim C_{n}$ can be chosen large enough so that on $G$,

$$
\begin{array}{ll}
R^{(n)}(t)=R(t)[ & (-1)^{n} \sum_{m=1}^{n} \sum_{\substack{|\alpha|=n \\
l(\alpha)=m}} d(\alpha) \sum_{j+1 \leq \ell_{1}, \cdots, \ell_{m} \leq k} \frac{1}{\left(t-w_{\ell_{1}}\right)^{\alpha_{1}}} \cdots \frac{1}{\left(t-w_{\ell_{m}}\right)^{\alpha_{m}}} \\
& \left.\quad+F_{n}(t)\right]
\end{array}
$$

where $\left|(d / d t)^{r} F_{n}(t)\right| \lesssim C_{n, r} A^{-1}|t|^{-n-r}$ for all $r \geq 0$. Here $\{d(\alpha)\}$ are combinatorial numbers defined on strictly positive multi-indices $\alpha$ such that the sums

$$
c_{m}(n)=\sum_{|\alpha|=n, l(\alpha)=m} d(\alpha)
$$

are the well-known Sterling numbers of the second kind; i.e., $\left\{c_{m}(n)\right\}_{m=1}^{n}$ are the coefficients of the polynomial

$$
x(x+1) \cdots(x+n-1)=\sum_{m=1}^{n} c_{m}(n) x^{m} .
$$


Proof. For $n=1$ we argue exactly as in Lemma 3.3, using (6) and (5) to obtain

$$
R^{\prime}(t)=R(t)\left[-\sum_{\ell=j+1}^{k} \frac{1}{t-w_{\ell}}+F_{1}(t)\right]
$$

where $F_{1}$ satisfies the appropriate derivative estimates on $G$. For general $n$ we argue by induction: if (7) holds for all derivatives up to order $n$,

$$
\begin{aligned}
& R^{(n+1)}(t) \\
& =R^{\prime}(t)\left[(-1)^{n} \sum_{m=1}^{n} \sum_{\substack{|\alpha|=n \\
l(\alpha)=m}} d(\alpha) \sum_{j+1 \leq \ell_{1}, \cdots, \ell_{m} \leq k} \frac{1}{\left(t-w_{\ell_{1}}\right)^{\alpha_{1}}} \cdots \frac{1}{\left(t-w_{\ell_{m}}\right)^{\alpha_{m}}}\right. \\
& \left.\quad+F_{n}(t)\right]+R(t)\left[(-1)^{n+1} \sum_{m=1}^{n} \sum_{\substack{|\alpha|=n \\
l(\alpha)=m}} d(\alpha) \sum_{r=1}^{m} \alpha_{r}\right. \\
& \left.\times \sum_{j+1 \leq \ell_{1}, \ldots, \ell_{m} \leq k} \frac{1}{\left(t-w_{\ell_{1}}\right)^{\alpha_{1}}} \cdots \frac{1}{\left(t-w_{\ell_{r}}\right)^{\alpha_{r}+1}} \cdots \frac{1}{\left(t-w_{\ell_{m}}\right)^{\alpha_{m}}}+F_{n}^{\prime}(t)\right] .
\end{aligned}
$$

Using (8) we obtain

$$
\begin{aligned}
R^{(n+1)}(t) & =R(t)\left[(-1)^{n+1} \sum_{m=1}^{n} \sum_{\substack{|\alpha|=n \\
l(\alpha)=m}} d(\alpha)\right. \\
& \times \sum_{\substack{j+1 \leq \ell_{1}, \cdots, \ell_{m}, \ell \leq k \\
l}} \frac{1}{\left(t-w_{\ell_{1}}\right)^{\alpha_{1}}} \cdots \frac{1}{\left(t-w_{\ell_{m}}\right)^{\alpha_{m}}} \frac{1}{t-w_{\ell}} \\
& +\quad \sum_{m=1}^{(-1)^{n+1} \sum_{|\alpha|=n, l(\alpha)=m}^{n}} d(\alpha) \sum_{r=1}^{m} \alpha_{r} \\
& \times \sum_{j+1 \leq \ell_{1}, \ldots, \ell_{m} \leq k} \frac{1}{\left(t-w_{\ell_{1}}\right)^{\alpha_{1}}} \cdots \frac{1}{\left(t-w_{\ell_{r}}\right)^{\alpha_{r}+1}} \cdots \frac{1}{\left(t-w_{\ell_{m}}\right)^{\alpha_{m}}}
\end{aligned}
$$

where

$$
\begin{aligned}
& F_{n+1}(t)=F_{n}(t)\left[-\sum_{\ell=j+1}^{k} \frac{1}{t-w_{\ell}}+F_{n}^{\prime}(t)\right] \\
& \quad+\left[(-1)^{n} F_{1}(t) \sum_{m=1}^{n} \sum_{|\alpha|=n, l(\alpha)=m} d(\alpha) \sum_{j+1 \leq \ell_{1}, \ldots, \ell_{m} \leq k} \frac{1}{\left(t-w_{\ell_{1}}\right)^{\alpha_{1}}} \cdots \frac{1}{\left(t-w_{\ell_{m}}\right)^{\alpha_{m}}}\right]
\end{aligned}
$$

satisfies the required derivative estimates on $G$.

Expressing $R^{(n+1)}(t)$ in the form (7) we see from (9) that the coefficients

$$
c_{m}(n+1)=\sum_{\substack{|\alpha|=n+1 \\ l(\alpha)=m}} d(\alpha)
$$


satisfy the recursive formulae:

$$
c_{n+1}(n+1)=1, \quad c_{k}(n+1)=n c_{k}(n)+c_{k-1}(n), k=1, \ldots, n
$$

where $c_{0}(n)=0$. These are the defining formulae for Sterling numbers of the second kind; the equivalent property for these numbers as the coefficients of the polynomial with roots at consecutive negative integers can be easily derived by induction:

$$
x(x+1) \cdots(x+n-1)=\sum_{k=1}^{n} c_{k}(n) x^{k}
$$

and so

$$
\begin{aligned}
x(x+1) \cdots(x+n) & =\sum_{k=1}^{n} c_{k}(n) x^{k+1}+\sum_{k=1}^{n} n c_{k}(n) x^{k} \\
& =n c_{1}(n) x+\sum_{k=2}^{n}\left(n c_{k}(n)+c_{k-1}(n)\right) x^{k}+x^{n+1} \\
& =\sum_{k=1}^{n+1} c_{k}(n+1) x^{k}
\end{aligned}
$$

by the above recursive formulae, completing the proof of Lemma 3.5.

Remark 3.6. The number of terms in the sum occurring in (7) is

$$
\begin{aligned}
\sum_{m=1}^{n} \sum_{\substack{|\alpha|=n \\
l(\alpha)=m}} d(\alpha)(k-j)^{m} & =\sum_{m=1}^{n} c_{m}(n)(k-j)^{m} \\
& =(k-j)(k-j+1) \cdots(k-j+n-1) .
\end{aligned}
$$

We now prove an extension of (6) and a generalisation of (5).

Lemma 3.7. Let $P, Q$ and $G$ be as in Lemmas 3.3 and 3.5 with $k \leq j$ (the completely analogous statement for $j \leq k$ also holds). Let $\mathcal{A}=\{\alpha\}$ and $\mathcal{B}=\{\beta\}$ be two $0(1)$ collections of strictly positive multi-indices of size $r ; r=|\alpha|=|\beta|$. Then on $G$, for $A \gtrsim 1$ large enough,

i) $\left|\left(t-z_{\ell_{1}}\right)^{-\alpha_{1}} \cdots\left(t-z_{\ell_{m}}\right)^{-\alpha_{m}}-\left(t-w_{\ell_{1}^{\prime}}\right)^{-\beta_{1}} \cdots\left(t-w_{\ell_{n}^{\prime}}\right)^{-\beta_{n}}\right| \lesssim A^{-1}|t|^{-r}$ for any $\alpha \in \mathcal{A}, \beta \in \mathcal{B}, r=\alpha_{1}+\cdots+\alpha_{m}=\beta_{1}+\cdots+\beta_{n}$, and $k+1 \leq$ $\ell_{1}, \ldots, \ell_{m}, \ell_{1}^{\prime}, \ldots, \ell_{n}^{\prime} \leq j$.

For each $\alpha \in \mathcal{A}$ associate a collection $\left\{z_{\ell_{1}}, \ldots, z_{\ell_{m}}\right\}$ where $m=l(\alpha)$ and $\ell_{1}, \ldots, \ell_{m} \leq j$. Then

ii) $\left|\sum_{\alpha \in \mathcal{A}}\left(t-z_{\ell_{1}}\right)^{-\alpha_{1}} \cdots\left(t-z_{\ell_{m}}\right)^{-\alpha_{m}}\right| \sim|t|^{-r}$.

Proof. The estimate in i) follows immediately from (6) by iteratively comparing factor by factor.

For ii), the upper bound follows easily from (5). For the lower bound, we use the fact $|z| \geq \operatorname{Re}(z)$ to see that the left hand side is larger than

$$
\left|\sum_{\alpha \in \mathcal{A}} \frac{\operatorname{Re} \prod_{s}\left(t-\bar{z}_{\ell_{s}}\right)^{\alpha_{s}}}{\prod_{s}\left|t-z_{\ell_{s}}\right|^{2 \alpha_{s}}}\right|=\left|\sum_{\alpha \in \mathcal{A}} \frac{R e\left[t^{r}+O\left(A^{-1} t^{r}\right)\right]}{|t|^{2 r}+O\left(A^{-1} t^{2 r}\right)}\right| \gtrsim|t|^{-r}
$$

for $A \gtrsim 1$ large enough, since $\left|z_{\ell}\right| \lesssim A^{-1}|t|$ on $G$ whenever $\ell \leq j$. 
In order to prove the decay estimate (3) of the Fourier transform of the measures defined in (2) we will use Lemmas 3.3 and 3.5 to estimate a determinant formed from the derivatives of the $n$ rational functions $R_{\ell}=P_{\ell} / Q_{\ell}, \ell=1, \ldots, n$, which make up the components of our curve $\Gamma=\left(R_{1}, \ldots, R_{n}\right)$. Here $P_{\ell}(t)=c_{\ell} \prod_{s=1}^{p_{\ell}}\left(t-z_{s}^{\ell}\right)$ and $Q_{\ell}(t)=d_{\ell} \prod_{s=1}^{q_{\ell}}\left(t-w_{s}^{\ell}\right)$ are polynomials with $\left|z_{1}^{\ell}\right| \leq \ldots \leq\left|z_{p_{\ell}}^{\ell}\right|$ and $\left|w_{1}^{\ell}\right| \leq \ldots \leq$ $\left|w_{q_{\ell}}^{\ell}\right|$ and the determinant of derivatives is

$$
\begin{aligned}
\triangle & :=\operatorname{det}\left(\Gamma^{\prime}(t), \ldots, \Gamma^{(n)}(t)\right) \\
& =\prod_{\ell=1}^{n} R_{\ell}(t) \sum_{\pi \in S_{n}}(-1)^{\operatorname{sgn}(\pi)}\left[R_{1}^{(\pi(1))} / R_{1}\right] \cdots\left[R_{n}^{(\pi(n))} / R_{n}\right]
\end{aligned}
$$

where $S_{n}$ denotes the group of permutations on $n$ letters. Let us define $\Lambda(t)$ to be the sum in the above formula so that $\triangle=\Lambda(t) \prod_{\ell} R_{\ell}(t)$.

For each $R_{\ell}$ consider a gap

$$
G_{\ell}=\left[A\left|z_{j_{\ell}}^{\ell}\right|, A^{-1}\left|z_{j_{\ell}+1}^{\ell}\right|\right] \cap\left[A\left|w_{k_{\ell}}^{\ell}\right|, A^{-1}\left|w_{k_{\ell}+1}^{\ell}\right|\right]
$$

and suppose $j_{\ell} \geq k_{\ell}$ when $1 \leq \ell \leq r$ and $k_{\ell}>j_{\ell}$ when $r+1 \leq \ell \leq n$, for some $0 \leq r \leq n$. The following lemma will estimate $\Lambda$ (and hence $\triangle$ by Lemma 3.1) on the intersection $G=\bigcap_{\ell=1}^{n} G_{\ell}$.

Lemma 3.8. Let $R_{\ell}=P_{\ell} / Q_{\ell}, \ell=1,2, \ldots, n, \Lambda$ and $G$ be as above and set $x_{\ell}=j_{\ell}-k_{\ell}$ for each $\ell$. Suppose that the integers $\left\{x_{\ell}\right\}_{\ell=1}^{n}$ are nonzero and distinct (so, in particular, $x_{\ell}>0,1 \leq \ell \leq r$ and $\left.x_{\ell}<0, r+1 \leq \ell \leq n\right)$. Then for $A \gtrsim 1$ large enough,

$$
\Lambda(t) \sim t^{-n(n+1) / 2}
$$

on $G$.

Proof. By Lemmas 3.3 and 3.5 we have

$$
\begin{array}{r}
\prod_{\ell=1}^{n}\left[R_{\ell}^{(\pi(\ell))} / R_{\ell}\right](t)=(-1)^{\sum_{s=r+1}^{n} \pi(s)} \sum_{\alpha \in E_{\pi}} \frac{1}{\left(t-u_{1, \alpha}\right)^{\alpha_{1}}} \cdots \frac{1}{\left(t-u_{m, \alpha}\right)^{\alpha_{m}}} \\
+O\left(A^{-1} t^{-n(n+1) / 2}\right)
\end{array}
$$

for each $\pi \in S_{n}$. Here $E_{\pi}$ is a collection of strictly positive multi-indices $\alpha$ with $|\alpha|=n(n+1) / 2$ whose cardinality, by Remarks 3.4 and 3.6, satisfies

$$
\begin{aligned}
\left|E_{\pi}\right|= & \prod_{\ell=0}^{\pi(1)-1}\left(x_{\pi(1)}-\ell\right) \cdots \prod_{\ell=0}^{\pi(r)-1}\left(x_{\pi(r)}-\ell\right) \\
& \times \prod_{\ell=0}^{\pi(r+1)-1}\left(-x_{\pi(r+1)}-\ell\right) \cdots \prod_{\ell=0}^{\pi(n)-1}\left(-x_{\pi(n)}-\ell\right) \\
= & (-1)^{\sum_{s=r+1}^{n} \pi(s)} \prod_{m=1}^{n} \prod_{\ell=0}^{\pi(m)-1}\left(x_{\pi(m)}-\ell\right) .
\end{aligned}
$$

The complex numbers $\left\{u_{\ell, \alpha}\right\}$ lie among the roots $\left\{z_{m}^{\ell}\right\}_{m=k_{\ell}+1}^{j_{\ell}} \cup\left\{w_{m}^{\ell}\right\}_{m=j_{\ell}+1}^{k_{\ell}}$.

The upper bound for $\Lambda$ on $G$ follows easily from Lemma 3.7, part ii). 
For the lower bound we split the permutations $\pi \in S_{n}$ into two families:

$$
S_{n, 1}=\left\{\pi \in S_{n}: \operatorname{sgn} \pi+\sum_{s=r+1}^{n} \pi(s) \text { is even }\right\}
$$

and

$$
S_{n, 2}=\left\{\pi \in S_{n}: \operatorname{sgn} \pi+\sum_{s=r+1}^{n} \pi(s) \text { is odd }\right\} .
$$

If we can show that

$$
\sum_{\pi \in S_{n, 1}}\left|E_{\pi}\right| \neq \sum_{\pi \in S_{n, 2}}\left|E_{\pi}\right|
$$

then we can pair off a term $\prod_{\ell=1}^{m}\left(t-u_{\ell, \alpha}\right)^{-\alpha_{\ell}}$ from $\alpha \in E_{\pi}$ and $\pi \in S_{n, 1}$ with a term $-\prod_{\ell=1}^{m}\left(t-u_{\ell, \beta}\right)^{-\beta_{\ell}}$ from $\beta \in E_{\pi}$ and $\pi \in S_{n, 2}$, using Lemma 3.7, part i), to achieve a bound $O\left(A^{-1} t^{-n(n+1) / 2}\right)$ in the sum defining $\Lambda$, showing that

$$
\Lambda(t)= \pm \sum_{\pi \in \mathcal{S}} \sum_{\alpha \in E_{\pi}} \frac{1}{\left(t-u_{1, \alpha}\right)^{\alpha_{1}}} \cdots \frac{1}{\left(t-u_{m, \alpha}\right)^{\alpha_{m}}}+O\left(A^{-1} t^{-n(n+1) / 2}\right)
$$

where $\mathcal{S}$ is either a nonempty subset of $S_{n, 1}\left(\right.$ when $\sum_{\pi \in S_{n, 1}}\left|E_{\pi}\right|>\sum_{\pi \in S_{n, 2}}\left|E_{\pi}\right|$ ) or a nonempty subset of $S_{n, 2}$ (when $\sum_{\pi \in S_{n, 2}}\left|E_{\pi}\right|>\sum_{\pi \in S_{n, 1}}\left|E_{\pi}\right|$ ). Lemma 3.7, part ii) can now be employed to show $\Lambda(t) \gtrsim t^{-n(n+1) / 2}$ on $G$ if $A \gtrsim 1$ is chosen large enough.

It remains therefore to establish (11). Observe that by (10),

$$
\sum_{\pi \in S_{n, 1}}\left|E_{\pi}\right|-\sum_{\pi \in S_{n, 2}}\left|E_{\pi}\right|=\operatorname{det}\left(\begin{array}{ccc}
x_{1} & \cdots & x_{n} \\
x_{1}\left(x_{1}-1\right) & & x_{n}\left(x_{n}-1\right) \\
\vdots & \ddots & \vdots \\
x_{1} \ldots\left(x_{1}-n+1\right) & & x_{n} \ldots\left(x_{n}-n+1\right)
\end{array}\right)
$$

and one can easily check that this determinant, call it $D_{n}$, is equal to

$$
x_{1} \ldots x_{n} \prod_{1 \leq r<s \leq n}\left(x_{s}-x_{r}\right)
$$

which is nonzero by our assumptions on the integers $\left\{x_{s}\right\}$. In fact, considering $D_{n}$ as a polynomial of degree $n$ in the variable $x_{n}$, we have

$$
D_{n}=p\left(x_{1}, \ldots, x_{n-1}\right)\left(x_{n}-x_{n-1}\right) \ldots\left(x_{n}-x_{1}\right) x_{n},
$$

since $D_{n}$, as a function of $x_{n}$, clearly vanishes at $x_{1}, x_{2}, \ldots, x_{n-1}$ and 0 . On the other hand, expanding $D_{n}$ in the last column shows that

$$
D_{n}=D_{n-1} x_{n}+\text { lower order terms in } x_{n}
$$

where $D_{n-1}$ is the analogous $(n-1) \times(n-1)$ determinant in the variables $x_{1}$, $x_{2}, \ldots, x_{n-1}$.

Hence by induction, we see that

$$
D_{n-1}=p\left(x_{1}, \ldots, x_{n-1}\right)=x_{1} \ldots x_{n-1} \prod_{1 \leq r<s \leq n-1}\left(x_{s}-x_{r}\right),
$$

establishing the claimed formula for $D_{n}$ by (12) and thus completing the proof of the lemma. 
Remark 3.9. The arguments used above show that $\left|\operatorname{det} A_{k}(t)(i \mid j)\right| \lesssim 1$, where $A_{k}(t)(i \mid j)$ is the $(n-1) \times(n-1)$ matrix formed by deleting the $i$-th row and the $j$-th column from

$$
A_{k}(t)=\left(\begin{array}{ccc}
2^{k} R_{1}^{\prime}\left(2^{k} t\right) 2^{-k x_{1}} & \cdots & 2^{k} R_{n}^{\prime}\left(2^{k} t\right) 2^{-k x_{n}} \\
\vdots & \ddots & \vdots \\
2^{n k} R_{1}^{(n)}\left(2^{k} t\right) 2^{-k x_{1}} & \cdots & 2^{n k} R_{n}^{(n)}\left(2^{k} t\right) 2^{-k x_{n}}
\end{array}\right),
$$

and where as in Lemma $3.8, x_{\ell}=j_{\ell}-k_{\ell}$.

We end this section by stating a very useful estimate for one dimensional oscillatory integrals, known as van der Corput's lemma. A proof can be found in $[6]$.

Lemma 3.10. Suppose that $\phi$ is real-valued and smooth on $(a, b)$, and that $\left|\phi^{(k)}(t)\right|$ $\geq \lambda>0$ for all $t \in(a, b)$. Then

$$
\left|\int_{a}^{b} e^{i \phi(t)} d t\right| \leq C_{k} \lambda^{-1 / k}
$$

holds when either $k \geq 2$ or $k=1$ and $\phi^{\prime}$ is monotone.

\section{BASIC REDUCTION}

In this section we will show that we can restrict our analysis of the operators $H$ and $M$, defined with respect to a curve $\Gamma=\left(R_{1}, \ldots, R_{n}\right)$ of rational functions, to an interval $G$ on which each $R_{\ell}(t) \sim c_{\ell} t^{j_{\ell}-k_{\ell}}$ where the numbers $\left\{j_{\ell}-k_{\ell}\right\}$ are nonzero and distinct. Furthermore there will be sufficient separation between appropriate roots of the polynomials defining $R_{\ell}$ to enable us to obtain derivative bounds as described in Lemmas 3.3 and 3.5 above. We will adopt the notation $R_{\ell}=P_{\ell} / Q_{\ell}$ for each $1 \leq \ell \leq n$ where $P_{\ell}(t)=\sum p_{j}^{\ell} t^{j}=C_{\ell} \prod\left(t-z_{j}^{\ell}\right)$ and the roots will always be ordered so that $\left|z_{1}^{\ell}\right| \leq\left|z_{2}^{\ell}\right| \leq \cdots$. Similarly $Q_{\ell}(t)=\sum q_{k}^{\ell} t^{k}=D_{\ell} \prod\left(t-w_{k}^{\ell}\right)$ where $\left|w_{1}^{\ell}\right| \leq\left|w_{2}^{\ell}\right| \leq \cdots$ for each $1 \leq \ell \leq n$. When we pass to transformed polynomials $\tilde{P}_{\ell}$ and $\tilde{Q}_{\ell}$, the notation for the transformed coefficients and roots is adjusted accordingly.

We begin with the singular integral operator $H$. Applying Lemma 3.1 to every denominator $Q_{\ell}$ of $R_{\ell}$ we can reduce the $L^{p}$ boundedness of $H$ to bounding

$$
H_{G} f(x)=\int_{|t| \in G} f\left(x_{1}-R_{1}(t), \ldots, x_{n}-R_{n}(t)\right) d t / t
$$

where $Q_{\ell} \sim c_{\ell} t^{k_{\ell}}$ on $G$ for every $1 \leq \ell \leq n$; see Remark 3.2. Here

$$
G \subset \bigcap_{\ell=1}^{n}\left[A\left|w_{k_{\ell}}^{\ell}\right|, A^{-1}\left|w_{k_{\ell}+1}^{\ell}\right|\right],
$$

which we assume is nonempty. In particular we have the following root separation between the roots of the polynomials $\left\{Q_{\ell}\right\}$; for any two roots $w_{k}^{\ell}$ and $w_{k^{\prime}}^{\ell^{\prime}}$ where $k \leq k_{\ell}$ and $k^{\prime}>k_{\ell^{\prime}}$,

$$
A\left|w_{k}^{\ell}\right| \leq A^{-1}\left|w_{k^{\prime}}^{\ell^{\prime}}\right|
$$

We could have applied Lemma 3.1 to each $P_{\ell}$ as well as $Q_{\ell}$ putting ourselves on an interval where $R_{\ell} \sim c_{\ell} t^{j_{\ell}-k_{\ell}}$, but the numbers $\left\{j_{\ell}-k_{\ell}\right\}$ would not necessarily be nonzero nor distinct. To get ourselves in the situation where the rational functions 
$\left\{R_{\ell}\right\}$ behave like monomials with powers which are nonzero and distinct, we will use the fact that the $L^{p}$ operator norm of $H_{G}$ is invariant under conjugation by the group of invertible affine transformations. This allows us to replace the curve $\Gamma=\left(R_{1}, \ldots, R_{n}\right)$ by $A \Gamma+\vec{v}$, where $A$ is any constant invertible $n \times n$ matrix and $\vec{v}$ is any constant vector in $\mathbb{R}^{n}$, without changing the operator norm of $H_{G}$.

We will apply $n$ affine transformations in succession, changing only one component of $\Gamma$ each time. The whole process will be carried out in $n$ steps. By the $r$ th step, we will have transformed the first $r-1$ components of $\Gamma, R_{\ell} \rightarrow \tilde{R}_{\ell}, 1 \leq$ $\ell \leq r-1$, and successfully reduced the analysis to a subinterval $G^{\prime} \subset G$ such that $\tilde{R}_{\ell}(t) \sim c_{\ell} t^{j_{\ell}^{\prime}-k_{\ell}^{\prime}}$ for $|t| \in G^{\prime}$ where $\left\{j_{\ell}^{\prime}-k_{\ell}^{\prime}\right\}$ are nonzero and distinct (furthermore, there will be sufficient root separation to guarantee higher derivative bounds for $\tilde{R}_{\ell}$ ). In the $r$ th step we will choose an appropriate affine transformation changing the $r$ th component $R_{r} \rightarrow \tilde{R}_{r}=\tilde{P}_{r} / \tilde{Q}_{r}$, leaving the other components alone, in such a way that certain coefficients of $\tilde{P}_{r}$ vanish. Then by employing Lemma 3.1, part ii), we will be able to remove $0(1)$ dyadic intervals from $G^{\prime}$ such that on each of the complementary intervals, $\tilde{R}_{r}(t) \sim c_{r} t_{r}^{j_{r}^{\prime}-k_{r}^{\prime}}$ where $j_{r}^{\prime}-k_{r}^{\prime}$ is nonzero and distinct from the numbers $j_{\ell}^{\prime}-k_{\ell}^{\prime}, 1 \leq \ell \leq r-1$ (higher derivative bounds will also hold). We will only need to annihilate coefficients of the transformed $\tilde{P}_{r}$ via the affine transformation since the process will transform the original denominators $\left\{Q_{r}\right\}$ into $\left\{\tilde{Q}_{r}=\prod_{\ell=1}^{r} Q_{\ell}\right\}$. Note that for each $1 \leq r \leq n, \tilde{Q}_{r}(t)=Q_{1} \cdots Q_{r}(t) \sim c t^{k_{1}+\cdots k_{r}}$ on $G$ because (13) implies

$$
\begin{aligned}
G & \subseteq \bigcap_{\ell=1}^{r}\left[A\left|w_{k_{\ell}}^{\ell}\right|, A^{-1}\left|w_{k_{\ell}+1}^{\ell}\right|\right] \\
& =\left[A \sup _{1 \leq \ell \leq r}\left|w_{k_{\ell}}^{\ell}\right|, A^{-1} \inf _{1 \leq \ell \leq r}\left|w_{k_{\ell}+1}^{\ell}\right|\right] \\
& =\left[A\left|w_{k_{1}+\cdots+k_{r}}\right|, A^{-1}\left|w_{k_{1}+\cdots+k_{r}+1}\right|\right]
\end{aligned}
$$

where $\left|w_{1}\right| \leq\left|w_{2}\right| \leq \cdots$ denote the roots of $\tilde{Q}_{r}=Q_{1} Q_{2} \ldots Q_{r}$.

We begin with the first step, using the affine transformation $I+\vec{v}$ with $\vec{v}=$ $(a, 0, \ldots, 0)$ to replace $R_{1}=P_{1} / Q_{1}$ with $\tilde{R}_{1}=R_{1}+a=\tilde{P}_{1} / Q_{1}$, where $\tilde{P}_{1}=$ $P_{1}+a Q_{1}=\sum \tilde{p}_{j}^{\prime} t^{j}$. Choose $a$ so that $\tilde{p}_{k_{1}}^{1}=p_{k_{1}}^{1}+a q_{k_{1}}^{1}=0$. By Lemma 3.1, part ii), if $\left|\tilde{z}_{1}^{1}\right| \leq\left|\tilde{z}_{2}^{1}\right| \leq \ldots$ denote the roots of $\tilde{P}_{1}$, the interval $\left[A\left|\tilde{z}_{k_{1}}^{1}\right|, A^{-1}\left|\tilde{z}_{k_{1}+1}^{1}\right|\right]$ is empty if $A \gtrsim 1$ is chosen large enough. Therefore we can decompose $G$ into gaps and dyadic intervals with respect to $\tilde{P}_{1}$ to reduce matters to bounding

$$
H_{G_{1}} f(x)=\int_{|t| \in G_{1}} f\left(x_{1}-\tilde{P}_{1} / Q_{1}, \ldots, x_{n}-P_{n} / Q_{n}\right) d t / t
$$

where $\tilde{P}_{1}(t) \sim \tilde{p}_{j_{1}^{\prime}}^{1} t^{j_{1}^{\prime}}$ on $G_{1} \subseteq G$ and $j_{1}^{\prime}-k_{1} \neq 0$.

Let us now suppose we have performed $r-1$ steps in the process reducing the analysis to bounding

$$
H_{G^{\prime}} f(x)=\int_{|t| \in G^{\prime}} f\left(x_{1}-\tilde{R}_{1}, \ldots, x_{r-1}-\tilde{R}_{r-1}, x_{r}-R_{r}, \ldots, x_{n}-R_{n}\right) d t / t
$$

where $\tilde{Q}_{\ell}(t)=\prod_{\mu=1}^{\ell} Q_{\mu}(t) \sim d_{\ell} t^{k_{\ell}^{\prime}}, k_{\ell}^{\prime}=k_{1}+\cdots+k_{\ell}$ and $\tilde{P}_{\ell}(t)=c_{\ell} t^{j_{\ell}^{\prime}}$ on $G^{\prime} \subseteq G$ so that the numbers $\left\{j_{\ell}^{\prime}-k_{\ell}^{\prime}\right\}$ are nonzero and distinct. The monomial behaviour 
of $\tilde{P}_{\ell}$ on $G^{\prime}$ is guaranteed by ensuring that we have the following root separation: if $\left|\tilde{z}_{1}^{\ell}\right| \leq\left|\tilde{z}_{2}^{\ell}\right| \leq \cdots$ denote the roots of $\tilde{P}_{\ell}$, then for any two roots $\tilde{z}_{j}^{\ell}$ and $\tilde{z}_{k}^{m}$ where $j \leq j_{\ell}^{\prime}$ and $k>j_{m}^{\prime}$,

$$
A\left|\tilde{z}_{j}^{\ell}\right| \leq A^{-1}\left|\tilde{z}_{k}^{m}\right|
$$

Recall that (14) gives us the analogous root separation for the polynomials $\left\{Q_{\ell}\right\}$. Furthermore we also have root separation between a root from $\tilde{z}_{j}^{\ell}, 1 \leq \ell \leq r-1$, and a root from $w_{k}^{\ell}, 1 \leq \ell \leq n$. We record here that the above root separation listed in (15) and (14) implies the following relationship between the coefficients of $\tilde{P}_{\ell}(t)=\sum \tilde{p}_{j}^{\ell} t^{j}$ and $Q_{\ell}(t)=\sum q_{k}^{\ell} t^{k}$ :

(16) if $\mu<j_{\ell}^{\prime}, \quad \tilde{p}_{\mu}^{\ell} \lesssim \tilde{p}_{j_{\ell}^{\prime}}^{\ell} \prod_{\sigma=\mu+1}^{j_{\ell}^{\prime}} \tilde{z}_{\sigma}^{\ell}, \quad$ and if $j_{\ell}^{\prime}<\mu, \quad \tilde{p}_{\mu}^{\ell} \lesssim \tilde{p}_{j_{\ell}^{\prime}}^{\ell} \prod_{\sigma=j_{\ell}^{\prime}+1}^{\mu}\left[\tilde{z}_{\sigma}^{\ell}\right]^{-1}$

for $1 \leq \ell \leq r-1$, and

(17) if $\mu<k_{\ell}, \quad q_{\mu}^{\ell} \lesssim q_{k_{\ell}}^{\ell} \prod_{\sigma=\mu+1}^{k_{\ell}} w_{\sigma}^{\ell}, \quad$ and if $k_{\ell}<\mu, \quad q_{\mu}^{\ell} \lesssim q_{k_{\ell}}^{\ell} \prod_{\sigma=k_{\ell}+1}^{\mu}\left[w_{\sigma}^{\ell}\right]^{-1}$

for $1 \leq \ell \leq n$. This is simply Lemma 3.1, part iii), applied to the polynomials $\left\{\tilde{P}_{\ell}\right\}$ and $\left\{Q_{\ell}\right\}$ and will play an important role in carrying out the $r$ th step.

The $r$ th step in the process uses the affine transform $A+\vec{v}$ where

$$
A=\left(\begin{array}{c|c|c}
\mathrm{I}_{r-1} & \multicolumn{2}{|c}{\mathrm{O}} \\
\hline a_{1} \ldots a_{r-1} & 1 & 0 \ldots 0 \\
\hline & \mathrm{O} & \mathrm{I}_{n-r}
\end{array}\right)
$$

and $\vec{v}=\left(0, \ldots, 0, a_{r}, 0, \ldots, 0\right)$ to transform $R_{r}$ to $\tilde{R}_{r}$, leaving the other components alone, where $\tilde{R}_{r}=\tilde{P}_{r} / \tilde{Q}_{r}, \tilde{Q}_{r}=\prod_{\ell=1}^{r} Q_{\ell}$ and

$$
\tilde{P}_{r}=a_{1} \tilde{P}_{1} \prod_{\ell=2}^{r} Q_{\ell}+\cdots+a_{r-1} \tilde{P}_{r-1} Q_{r}+a_{r} \prod_{\ell=1}^{r} Q_{\ell}+P_{r} \prod_{\ell=1}^{r-1} Q_{\ell} .
$$

The idea is to choose $a_{1}, a_{2}, \ldots, a_{r}$ so that the $j_{\ell}^{\prime}-k_{\ell}^{\prime}+k_{r}^{\prime}, 1 \leq \ell \leq r-1$, and $k_{r}^{\prime}$ th coefficients of $\tilde{P}_{r}$ vanish (here $k_{\ell}^{\prime}=k_{1}+\cdots+k_{\ell}$ ). Therefore we need to find a solution $\vec{a}=\left(a_{1}, \ldots, a_{r}\right)$ to $S \vec{a}=-\left(\sigma_{1}, \ldots, \sigma_{r}\right)$,

$$
S=\left(\begin{array}{ccc}
\sigma_{1}^{1} & \cdots & \sigma_{r}^{1} \\
\vdots & \ddots & \vdots \\
\sigma_{1}^{r} & \cdots & \sigma_{r}^{r}
\end{array}\right)
$$

where for $1 \leq \ell \leq r-1, \sigma_{m}^{\ell}$ is the $\left(j_{\ell}^{\prime}-k_{\ell}^{\prime}+k_{r}^{\prime}\right)$ th coefficient of $\tilde{P}_{m} Q_{m+1} \cdots Q_{r}$, when $1 \leq m \leq r-1$, and of $Q_{1} \cdots Q_{r}$, when $m=r$. For $\ell=r, \sigma_{m}^{r}$ is the $k_{r}^{\prime}$ th coefficient of $\tilde{\tilde{P}}_{m} Q_{m+1} \cdots Q_{r}$, when $1 \leq m \leq r-1$, and of $Q_{1} \cdots Q_{r}$, when $m=r$. Finally, $\sigma_{\ell}$ is the $\left(j_{\ell}^{\prime}-k_{\ell}^{\prime}+k_{r}^{\prime}\right)$ th (when $1 \leq \ell \leq r-1$ ) and $k_{r}^{\prime}$ th (when $\ell=r$ ) coefficient of $P_{r} Q_{1} \cdots Q_{r-1}$. This is accomplished by showing

$$
\operatorname{det} S \neq 0
$$

when $A \gtrsim 1$ is chosen large enough in (14) and (15). 
Remarks 4.1.

- The case $r=1$ of (18) where we consider just one polynomial says that if the $k$ th root is separated from the $(k+1)$ th root, then the $k$ th coefficient of the polynomial must be nonzero. This is just Lemma 3.1, part (ii), which we have used extensively.

- The special case $r=2$ of (18), where one takes $Q_{1}=1, P_{1}=t$ and $Q_{2}$ an arbitrary polynomial was considered in [3].

If (18) holds, then we can successfully solve $S \vec{a}=-\left(\sigma_{1}, \ldots, \sigma_{r}\right)$ guaranteeing that the appropriate coefficients of $\tilde{P}_{r}$ vanish and hence, by Theorem 3.1, part ii), if $\left|\tilde{z}_{1}^{r}\right| \leq\left|\tilde{z}_{2}^{r}\right| \leq \cdots$ denote the roots of $\tilde{P}_{r}$, the intervals $\left[A\left|\tilde{z}_{j}^{r}\right|, A^{-1}\left|\tilde{z}_{j+1}^{r}\right|\right]$ are empty whenever $j \in\left\{j_{\ell}^{\prime}-k_{\ell}^{\prime}+k_{r}^{\prime}\right\}_{\ell=1}^{r-1}$ or $j=k_{r}^{\prime}$ if $A \gtrsim 1$ is chosen large enough. Therefore we can decompose $G^{\prime}$ into $0(1)$ gaps and dyadic intervals with respect to $\tilde{P}_{r}$ to reduce matters to bounding

$$
H_{G^{\prime \prime}} f(x)=\int_{|t| \in G^{\prime \prime}} f\left(x_{1}-\tilde{R}_{1}, \cdots, x_{r}-\tilde{R}_{r}, \cdots, x_{n}-R_{n}\right) d t / t
$$

where $\tilde{R}_{r}(t) \sim c_{r} t^{j_{r}^{\prime}-k_{r}^{\prime}}$ for $|t| \in G^{\prime \prime}$ and $j_{r}^{\prime} \notin\left\{j_{\ell}^{\prime}-k_{\ell}^{\prime}+k_{r}^{\prime}\right\}_{\ell=1}^{r-1}$ and $j_{r}^{\prime} \neq k_{r}^{\prime}$, completing the $r$ th step of the process. It remains to establish (18).

First of all,

$$
\begin{aligned}
\operatorname{det} S & =\sum_{\pi \in S_{r}}(-1)^{\operatorname{sgn}(\pi)} \sigma_{1}^{\pi(1)} \cdots \sigma_{r}^{\pi(r)} \\
& =\sigma_{1}^{1} \cdots \sigma_{r}^{r}+\sum_{\pi \neq i d}(-1)^{\operatorname{sgn}(\pi)} \sigma_{1}^{\pi(1)} \cdots \sigma_{r}^{\pi(r)}
\end{aligned}
$$

For $1 \leq m \leq r-1, \sigma_{m}^{m}$ is the $N_{m}$ th coefficient of $\tilde{P}_{m} Q_{m+1} \cdots Q_{r}$ where $N_{m}=$ $j_{m}^{\prime}-k_{m}^{\prime}+k_{r}^{\prime}=j_{m}^{\prime}+k_{m+1}+\cdots+k_{r}$ and so

$$
\begin{aligned}
& \sigma_{m}^{m}=\sum_{\substack{\left(\ell, \ell_{1}, \cdots, \ell_{r-m}\right): \\
\ell+\ell_{1}+\cdots+\ell_{r-m}=N_{m}}} \tilde{p}_{\ell}^{m} q_{\ell_{1}}^{m+1} q_{\ell_{2}}^{m+2} \cdots q_{\ell_{r-m}}^{r} \\
& =\tilde{p}_{j_{m}^{\prime}}^{m} q_{k_{m+1}}^{m+1} \cdots q_{k_{r}}^{r}+\sum \quad \tilde{p}_{\ell}^{m} q_{\ell_{1}}^{m+1} q_{\ell_{2}}^{m+2} \cdots q_{\ell_{r-m}}^{r} . \\
& \left(\ell, \ell_{1}, \cdots, \ell_{r-m}\right) \neq\left(j_{m}^{\prime}, k_{m+1}, \cdots, k_{r}\right) \text { : } \\
& \ell+\ell_{1}+\cdots+\ell_{r-m}=N_{m}
\end{aligned}
$$

Fix $\left(\ell, \ell_{1}, \ldots, \ell_{r-m}\right) \neq\left(j_{m}^{\prime}, k_{m+1}, \ldots, k_{r}\right)$ satisfying $\ell+\ell_{1}+\cdots+\ell_{r-m}=N_{m}$, and set $n_{s}=k_{s}-\ell_{s-m}$ if $m+1 \leq s \leq r, s \neq r, n_{r}=j_{m}^{\prime}-\ell$. Therefore $\sum_{s=m}^{r} n_{s}=0$. Furthermore if $E_{+}=\left\{m \leq s \leq r: n_{s} \geq 0\right\}$ and $E_{-}=\left\{m \leq s \leq r: n_{s}<0\right\}$,

$$
-\sum_{s \in E_{-}} n_{s}=\sum_{s \in E_{+}} n_{s}>0
$$

Without loss of generality assume that $m \in E_{+}$. Then (16) implies that $\tilde{p}_{\ell}^{m} \lesssim$ $\tilde{p}_{j_{m}^{\prime}}^{m} \prod_{\sigma=\ell+1}^{j_{m}^{\prime}} \tilde{z}_{\sigma}^{m}$. If $s \in E_{+} \backslash\{r\}$, then (17) implies $q_{\ell_{s-m}}^{s} \lesssim q_{k_{s}}^{s} \prod_{\sigma=\ell_{s-m}+1}^{k_{s}} w_{\sigma}^{s}$ and 
if $s \in E_{-}$, then $q_{\ell_{s-m}}^{s} \lesssim q_{k_{s}}^{s} \prod_{\sigma=k_{s}+1}^{\ell_{s-m}}\left[w_{\sigma}^{s}\right]^{-1}$. Hence

$$
\begin{aligned}
\tilde{p}_{\ell}^{m} \prod_{s=m+1}^{r} q_{\ell_{s-m}}^{s} \lesssim \tilde{p}_{j_{m}^{\prime}}^{m} & \prod_{s=m+1}^{r} q_{k_{s}}^{s} \times \prod_{\sigma=\ell+1}^{j_{m}^{\prime}} \tilde{z}_{\sigma}^{m} \prod_{s \in E_{+} \backslash\{r\}} \prod_{\sigma=\ell_{s-m}+1}^{k_{s}} w_{\sigma}^{s} \\
& \times \prod_{s \in E_{-}} \prod_{\sigma=k_{s}+1}^{\ell_{s-m}}\left[w_{\sigma}^{s}\right]^{-1} .
\end{aligned}
$$

The last two products are nonempty and both products contain exactly the same number of terms, $-\sum_{s \in E_{-}} n_{s}=\sum_{s \in E_{+}} n_{s}>0$. For any term in the first product, say $w_{\sigma}^{s}$ with $s \in E_{+} \backslash\{r\}$, and any term in the second product, say $\left[w_{\sigma^{\prime}}^{s^{\prime}}\right]^{-1}$ with $s^{\prime} \in E_{-}$, we have $w_{\sigma}^{s}\left[w_{\sigma^{\prime}}^{s^{\prime}}\right]^{-1} \lesssim A^{-1}$ by (14), (15) and the remark which follows these estimates. Hence $\tilde{p}_{\ell}^{m} q_{\ell_{1}}^{m+1} \cdots q_{\ell_{r-m}}^{r} \lesssim A^{-1} \tilde{p}_{j_{m}^{\prime}}^{m} q_{k_{m+1}}^{m+1} \cdots q_{k_{r}}^{r}$ and so by (19),

$$
\sigma_{m}^{m} \sim \tilde{p}_{j_{m}^{\prime}}^{m} q_{k_{m+1}}^{m+1} \cdots q_{k_{r}}^{r}
$$

for $1 \leq m \leq r-1$ if $A \gtrsim 1$ is large enough. Similarly $\sigma_{r}^{r} \sim q_{k_{r}}^{r}$ and therefore

$$
\sigma_{1}^{1} \cdots \sigma_{r}^{r} \sim q_{k_{r}}^{r} \prod_{m=1}^{r-1}\left[\tilde{p}_{j_{m}^{\prime}}^{m} q_{k_{m+1}}^{m+1} \cdots q_{k_{r}}^{r}\right] .
$$

Therefore (18) will be established once we prove that for every $\pi \neq i d$,

$$
\sigma_{1}^{\pi(1)} \cdots \sigma_{r}^{\pi(r)} \lesssim A^{-1} q_{k_{r}}^{r} \prod_{m=1}^{r-1}\left[\tilde{p}_{j_{m}^{\prime}}^{m} q_{k_{m+1}}^{m+1} \cdots q_{k_{r}}^{r}\right] .
$$

For notational convenience we will suppose that $\pi$ is a permutation on $\{1, \ldots$, $r-1\}$, that is, $\pi(r)=r$ (the other cases are similar). Then

$$
\sigma_{1}^{\pi(1)} \cdots \sigma_{r-1}^{\pi(r-1)}=\sum_{\substack{\left(\ell_{s}, \ell_{s, 1}, \cdots, \ell_{s, r-s}, 1 \leq s<r: \\ \ell_{s}+\ell_{s, 1}+\cdots+\ell_{s, r-s}=N_{\pi(s)}\right.}} \prod_{m=1}^{r-1}\left[\tilde{p}_{\ell_{m}}^{m} q_{\ell_{m, 1}}^{m+1} \cdots q_{\ell_{m, r-m}}^{r}\right]
$$

where $N_{\pi(s)}=j_{\pi(s)}^{\prime}+k_{\pi(s)+1}+\cdots+k_{r}$ as before. For each $1 \leq s \leq r-1$, fix an $(r-s+1)$-tuple $\left(\ell_{s}, \ell_{s, 1}, \ldots, \ell_{s, r-s}\right)$ so that $\ell_{s}+\ell_{s, 1}+\cdots+\ell_{s, r-s}=N_{\pi(s)}$ and set

$$
n_{t}^{(s)}= \begin{cases}\ell_{s, t-s}-k_{t}, & s+1 \leq t \leq r \\ \ell_{s}-j_{s}^{\prime}, & t=s\end{cases}
$$

Define $E_{+}=\left\{(s, t): n_{t}^{(s)} \geq 0\right\}$ and $E_{-}=\left\{(s, t): n_{t}^{(s)}<0\right\}$. If

$$
-\sum_{(s, t) \in E_{-}} n_{t}^{(s)}=\sum_{(s, t) \in E_{+}} n_{t}^{(s)}>0
$$

we can argue exactly as above to see that

$$
\prod_{s=1}^{r-1}\left[\tilde{p}_{\ell_{s}}^{s} q_{\ell_{s, 1}}^{s+1} \cdots q_{\ell_{s, r-s}}^{r}\right] \lesssim A^{-1} \prod_{s=1}^{r-1}\left[\tilde{p}_{j_{s}^{\prime}}^{s} q_{k_{s}+1}^{s+1} \cdots q_{k_{r}}^{r}\right] .
$$

This together with $\sigma_{r}^{r} \sim q_{k_{r}}^{r}$, proved previously, gives us (20) and therefore (18). It remains to prove $(21)$. 
For each $1 \leq s \leq r-1$,

$$
\sum_{t=s}^{r} n_{t}^{(s)}=\ell_{s}+\ell_{s, 1}+\cdots+\ell_{s, r-s}-\left(j_{s}^{\prime}+k_{s+1}+\cdots+k_{r}\right)
$$

and so summing over $1 \leq s \leq r-1$ shows that $\sum_{(s, t)} n_{t}^{(s)}=0$ since $\pi$ is assumed to be a permutation of $\{1, \ldots, r-1\}$. Hence

$$
-\sum_{(s, t) \in E_{-}} n_{t}^{(s)}=\sum_{(s, t) \in E_{+}} n_{t}^{(s)}
$$

and therefore, if $\sum_{(s, t) \in E_{+}} n_{t}^{(s)}=0, n_{t}^{(s)}=0$ for all $(s, t)$ implying that $\ell_{s}=j_{s}^{\prime}$ and $\ell_{s, m}=k_{s, m}, 1 \leq m \leq r-s$ for each $1 \leq s \leq r-1$. In particular, $N_{s}=N_{\pi(s)}$ for all $1 \leq s \leq r-1$. But $\pi \neq i d$ implies there exists an $1 \leq s_{0} \leq r-1$ such that $\pi\left(s_{0}\right) \neq s_{0}$, which in turn implies that $N_{\pi\left(s_{0}\right)} \neq N_{s_{0}}$ since the numbers $\left\{j_{s}^{\prime}-k_{s}^{\prime}\right\}$ are distinct. This proves (21) and thus finishes the proof of (20) and hence (18), completing the basic reduction for $H$.

A similar procedure allows us to reduce the study of the maximal function $M$ to

$$
M_{G} f(x)=\sup _{k}\left|2^{-k} \int_{t \in\left[2^{k}, 2^{k+1}\right] \cap G} f(x-\Gamma(t)) d t\right|
$$

where each component $R_{\ell}$ of $\Gamma$ satisfies $R_{\ell}(t) \sim c_{\ell} t^{j_{\ell}-k_{\ell}}$ on $G$, together with higher order derivative bounds, and where the exponents $\left\{j_{\ell}-k_{\ell}\right\}$ are nonzero and distinct.

\section{Proof of Theorem 1.1}

The previous section described a procedure which reduces the proof of Theorem 1.1 to establishing $L^{p}$ bounds, $1<p<\infty$, for

$$
\begin{aligned}
& H_{G} f(x)=\int_{|t| \in G} f\left(x_{1}-R_{1}(t), \ldots, x_{n}-R_{n}(t)\right) d t / t \\
= & \sum_{k} \int_{|t| \in G \cap\left[2^{k}, 2^{k+1}\right]} f\left(x_{1}-R_{1}(t), \ldots, x_{n}-R_{n}(t)\right) d t / t
\end{aligned}
$$

and

$$
M_{G} f(x)=\sup _{k}\left|2^{-k} \int_{t \in G \cap\left[2^{k}, 2^{k+1}\right]} f\left(x_{1}-R_{1}(t), \ldots, x_{n}-R_{n}(t)\right) d t\right|
$$

where $R_{\ell}(t) \sim c_{\ell} t^{j_{\ell}-k_{\ell}}$ on $G$, together with appropriate higher derivative bounds, and where the exponents $\left\{L_{\ell}=j_{\ell}-k_{\ell}\right\}$ are nonzero and distinct. By normalising the integration in the above sums defining $H_{G}$ and $M_{G}$ we may express

$$
H_{G} f(x)=\sum_{k} f * \sigma_{k}^{(k)}(x) \quad \text { and } \quad M_{G} f(x)=\sup _{k}\left|f * \mu_{k}^{(k)}(x)\right|
$$

where $\sigma_{k}^{(k)}$ and $\mu_{k}^{(k)}$ are $2^{k}$ dilates, $2^{k} \circ x=\left(2^{L_{1} k} x_{1}, \ldots, 2^{L_{n} k} x_{n}\right)$, of

$$
\left\langle\sigma^{(k)}, \phi\right\rangle:=\int_{|t| \in 2^{-k} G \cap[1,2]} \phi\left(\Gamma^{(k)}(t)\right) d t / t
$$

and

$$
\left\langle\mu^{(k)}, \phi\right\rangle:=\int_{t \in 2^{-k} G \cap[1,2]} \phi\left(\Gamma^{(k)}(t)\right) d t .
$$


Here $\Gamma^{(k)}(t)=\left(R_{1}^{(k)}(t), \ldots, R_{n}^{(k)}(t)\right)$ is a normalised curve so that

$$
R_{\ell}^{(k)}(t):=2^{-L_{\ell} k} R_{\ell}\left(2^{k} t\right) \sim 1 \quad \text { on } 2^{-k} G \cap[1,2]
$$

for each $1 \leq \ell \leq n$. As outlined in section 2 , see (3), our first goal is to prove the regularity estimates

$$
\left|\widehat{\sigma^{(k)}}(\xi)\right|,\left|\widehat{\mu^{(k)}}(\xi)\right| \leq C|\xi|^{-\epsilon}
$$

for some $\epsilon>0$, independent of $k$. We concentrate on establishing (24) for

$$
\widehat{\mu^{(k)}}(\xi)=\int_{t \in[1,2] \cap 2^{-k} G} e^{i\left[\xi_{1} R_{1}^{(k)}(t)+\cdots+\xi_{n} R_{n}^{(k)}(t)\right]} d t,
$$

and we will do so as an application of Lemmas 3.8 and 3.10. Let $\phi(t)=\xi_{1} R_{1}^{(k)}(t)+$ $\cdots+\xi_{n} R^{(k)}(t)=\xi \cdot \Gamma_{k}(t)$ be the phase in the above oscillatory integral defining $\widehat{\mu^{(k)}}$ and consider the vector of derivatives $\vec{d}(t)=\left(\phi^{\prime}(t), \ldots, \phi^{(n)}(t)\right)=A_{k}(t) \xi$ where

$$
A_{k}(t)=\left(\begin{array}{ccc}
2^{k}(d / d t) R_{1}^{(k)}(t) & \cdots & 2^{k}(d / d t) R_{n}^{(k)}(t) \\
\vdots & \ddots & \vdots \\
2^{n k}(d / d t)^{n} R_{1}^{(k)}(t) & \cdots & 2^{n k}(d / d t)^{n} R_{n}^{(k)}(t)
\end{array}\right) .
$$

Note that

$$
\operatorname{det} A_{k}(t)=2^{\frac{n(n+1)}{2} k} \Lambda\left(2^{k} t\right) \prod_{\ell=1}^{n} R_{\ell}^{(k)}(t)
$$

where $\Lambda$ is defined in section 3 prior to the statement of Lemma 3.8, which shows that $\Lambda\left(2^{k} t\right) \sim 2^{-\frac{n(n+1)}{2} k}$ on $2^{-k} G \cap[1,2]$ and so, together with (23), we have $\operatorname{det} A_{k}(t) \sim 1$ on $2^{-k} G \cap[1,2]$. Furthermore by Remark 3.9, all the cofactors $A_{k}(t)(i \mid j)$ of $A_{k}(t)$ are bounded above and so $\left\|\left[A_{k}(t)\right]^{-1}\right\| \lesssim 1$, implying

$$
|\xi| \leq\left\|\left[A_{k}(t)\right]^{-1}\right\||\vec{d}(t)| \lesssim|\vec{d}(t)| \lesssim\left|\phi^{\prime}(t)\right|+\cdots+\left|\phi^{(n)}(t)\right|
$$

for $t \in 2^{-k} G \cap[1,2]$.

We are now in a position to prove $(24)$ for $\widehat{\mu^{(k)}}(\xi)$ via van der Corput's lemma, Lemma 3.10, but in order to do this, we first split the interval $[1,2] \cap 2^{-k} G=\bigcup I$ into $0(1)$ subintervals such that on each subinterval $I$ we have

- $\left|\phi^{(r)}(t)\right|=\max \left\{\left|\phi^{\prime}(t)\right|, \ldots,\left|\phi^{(n)}(t)\right|\right\}$ for some $1 \leq r \leq n$ and

- $\phi^{\prime}$ is monotone.

On each subinterval $I$ we apply van der Corput's lemma and obtain (24) with $\epsilon=1 / n$ for $\widehat{\mu^{(k)}}$. A similar argument shows that $(24)$ holds for $\widehat{\sigma^{(k)}}$.

As discussed in section 2 we are unable to use the general results of Ricci and Stein in [4] to complete the proof of Theorem 1.1 since our measures $\left\{\mu_{k}^{(k)}\right\}$ and $\left\{\sigma_{k}^{(k)}\right\}$ defining $M_{G}$ and $H_{G}$ in (22) do not satisfy the further regularity condition that they are uniformly dominated by a fixed positive finite measure. Such a regularity condition implies $\left\|\mu_{k}^{(k)}\right\|,\left\|\sigma_{k}^{(k)}\right\| \lesssim 1$ and hence

$$
\left|\widehat{\mu^{(k)}}(\xi)-\widehat{\mu^{(k)}}(0)\right|, \quad\left|\widehat{\sigma^{(k)}}(\xi)\right| \lesssim|\xi|,
$$

complementing the decay estimates (24). These estimates in fact do hold in our case and follow immediately from (23). We will use (24) and (26) and their variants, together with an iteration of certain results for 1-parameter maximal and singular integral operators established by Duoandikoetxea and Rubio de Francia in [2], to 
prove Theorem 1.1. For the maximal function $M_{G}$ we use the following general result in $[2]$ which we now describe.

Let $\left\{a_{k}\right\}$ be a lacunary sequence of positive numbers; that is, $\inf _{k} a_{k} / a_{k+1}>1$ or $\inf _{k} a_{k+1} / a_{k}>1$. Decompose $\mathbb{R}^{n}=\mathbb{R}^{m} \times \mathbb{R}^{n-m}$ with $1 \leq m<n$ and write $x \in \mathbb{R}^{n}$ in the form $x=\left(x^{0}, \bar{x}\right) \in \mathbb{R}^{m} \times \mathbb{R}^{n-m}$. For any finite measure $\mu$ on $\mathbb{R}^{n}$ and Borel set $E \subseteq \mathbb{R}^{m}$ set $\mu^{(0)}(E)=\mu\left(E \times \mathbb{R}^{n-m}\right)$ which defines a measure on $\mathbb{R}^{m}$. We now state Theorem $\mathrm{C}$ in [2].

Proposition 5.1. Let $\left\{\mu_{k}\right\}$ be a sequence of positive measures on $\mathbb{R}^{n},\left\|\mu_{k}\right\| \lesssim 1$, such that

$$
\begin{gathered}
\left|\widehat{\mu_{k}}\left(\xi^{0}, \bar{\xi}\right)-\widehat{\mu_{k}}\left(\xi^{0}, 0\right)\right| \leq C\left|a_{k+1} \bar{\xi}\right|^{\delta} \\
\left|\widehat{\mu_{k}}\left(\xi^{0}, \bar{\xi}\right)\right| \leq C\left|a_{k} \bar{\xi}\right|^{-\delta}
\end{gathered}
$$

for some $\delta>0$. Suppose that $M^{0} g\left(x^{0}\right)=\sup _{k}\left|\mu_{k}^{(0)} * g\left(x^{0}\right)\right|$ is a bounded operator on $L^{p}\left(\mathbb{R}^{m}\right)$ for all $p>1$. Then $M f(x)=\sup _{k}\left|\mu_{k} * f(x)\right|$ is also bounded on $L^{p}\left(\mathbb{R}^{n}\right)$ for all $p>1$.

We apply this proposition to the sequence of positive measures $\left\{\mu_{k}^{(k)}\right\}$ defining $M_{G}$ in $(22)$ with $\xi^{0}=\left(\xi_{1}, \ldots, \xi_{n-1}\right), \bar{\xi}=\xi_{n}$ and $a_{k}=2^{k L_{n}}$, which satisfies the lacunarity condition since $L_{n} \neq 0$. The estimates (27) and (28) follow from (23) and (24). Hence Proposition 5.1 reduces matters to bounding the maximal operator

$$
M^{0} f\left(x^{0}\right)=\sup _{k}\left|2^{-k} \int_{\left[2^{k}, 2^{k+1}\right] \cap G} f\left(x_{1}^{0}-R_{1}(t), \ldots, x_{n-1}^{0}-R_{n-1}(t)\right) d t\right|,
$$

which effectively lowers the dimension by one. The analogues of (27) and (28) for $M^{0}$ are easily checked to be satisfied, and therefore we can apply Proposition 5.1 to $M^{0}$, further lowering the dimension by one, and iterating $n$ times successfully bounds $M_{G}$ on all $L^{p}, 1<p \leq \infty$.

The argument for $H_{G} f(x)=\sum_{k} f * \sigma_{k}^{(k)}(x)$ is slightly more involved. Here we will use Theorem $\mathrm{D}^{\prime}$ in [2].

Proposition 5.2. Let $\left\{\sigma_{k}\right\}$ be a sequence of measures on $\mathbb{R}^{n}$ such that $\left\|\sigma_{k}\right\| \lesssim 1$, satisfying

$$
\left|\widehat{\sigma_{k}}\left(\xi^{0}, \bar{\xi}\right)\right| \leq C \min \left(\left|a_{k+1} \bar{\xi}\right|,\left|a_{k} \bar{\xi}\right|^{-1}\right)^{\delta}
$$

for some $\delta>0$. Suppose that $\sigma^{*}(f)=\sup _{k}|| \sigma_{k}|* f|$ and $\sigma_{0}^{*}(f)=\sup _{k}|| \sigma_{k}^{0}|* f|$ are bounded operators on $L^{p}\left(\mathbb{R}^{n}\right)$ and $L^{q}\left(\mathbb{R}^{m}\right)$ respectively. Then $T f=\sum_{k} \sigma_{k} * f$ is bounded on $L^{p}\left(\mathbb{R}^{n}\right)$ for all $p$ satisfying $\left|\frac{1}{p}-\frac{1}{2}\right|<\frac{1}{2 q}$.

For notational convenience we write $\sigma_{k}$ for the measures $\sigma_{k}^{(k)}$ defining $H_{G}$ in (22). We will initially apply Proposition 5.2 with $m=n-1, \xi^{0}=\left(\xi_{1}, \ldots, \xi_{n-1}\right)$ and $\bar{\xi}=\xi_{n}$, but first we decompose $\sigma_{k}=\sigma_{k, 1}+\sigma_{k, 2}$, where $\sigma_{k, 2}=\sigma_{k}^{(0)} \otimes \phi_{k}$. Here $\sigma_{k}^{(0)}$ is defined analogously to $\mu_{k}^{(0)}$ above, and $\phi_{k}$ is an $L^{1}$-normalised $\left(\widehat{\phi_{k}}\left(\xi_{n}\right)=\widehat{\phi}\left(2^{k L_{n}} \xi_{n}\right)\right)$ positive Schwartz function on $\mathbb{R}$ with integral 1 and $\sigma_{k, 1}=\sigma_{k}-\sigma_{k, 2}$.

From (24) and (23), we see that $\sigma_{k, 1}$ satisfies (29) with $a_{k}=2^{L_{n} k}$. Furthermore the maximal functions $\sigma^{*}$ and $\sigma_{(0)}^{*}$ corresponding to the measures $\left\{\sigma_{k, 1}\right\}$ are bounded on all $L^{q}, 1<q<\infty$, by the same arguments for $M_{G}$, and so Proposition 5.2 applies to $\left\{\sigma_{k, 1}\right\}$, showing $f \rightarrow \sup _{k}\left|f * \sigma_{k, 1}\right|$ is bounded on all $L^{p}, 1<p<\infty$, leaving us to bound $f \rightarrow \sup _{k}\left|f * \sigma_{k, 2}\right|$. We have effectively reduced the dimension 
by one and applying the same argument to $f \rightarrow \sup _{k}\left|f * \sigma_{k, 2}\right|$ reduces another dimension. Iterating this procedure $n$ times establishes the desired $L^{p}$ bounds for $H_{G}$, completing the proof of Theorem 1.1.

\section{REFERENCES}

[1] A. Carbery, F. Ricci, J. Wright, Maximal functions and Hilbert transforms associated to polynomials, Revista Mat. Ibero. 14 (1998), 117-144. MR1639291 (99k:42014)

[2] J. Duoandikoetxea, José L. Rubio de Francia, Maximal and singular operators via Fourier transform estimates, Invent. Math. 84 (1986), 541-561 MR837527 (87f:42046)

[3] M. Folch-Gabayet, J. Wright, An oscillatory integral estimate associated to rational phases, J. Geom. Anal. 13 (2003), 291-299. MR1967028 (2004b:42025)

[4] F. Ricci, E.M. Stein, Multiparameter singular integrals and maximal functions, Ann. Inst. Fourier (Grenoble) 42 (1992), 637-670. MR1182643 (94d:42020)

[5] E.M. Stein, S. Wainger, Problems in harmonic analysis related to curvature, Bull. Amer. Math. Soc. 84 (1978), 1239-1295. MR508453 (80k:42023)

[6] E.M. Stein, Harmonic Analysis, Princeton Univ. Press (1993). MR1232192 (95c:42002)

Instituto de Matemáticas, UnAM, Area de la Investigación Científica, Circuito Exterior, Ciudad Universitaria, MÉxico, D.F. 04510

E-mail address: folchgab@matem.unam.mx

School of Mathematics, University of Edinburgh, JCMB, King's Buildings, Mayfield RoAd, Edinburgh EH9 3JZ, Scotland

E-mail address: j.r.wright@ed.ac.uk 